\title{
EXCITATION CLASS OF NEBULAE AS AN EVOLUTION CRITERION
}

\author{
G.A. GURZADYAN and A.G. EGIKYAN \\ Garny Space Astronomy Institute, Armenia
}

\begin{abstract}
A principally new, quantitative system of the classification of spectra of planetary nebulae is proposed. The excitation class of a nebula, $\mathrm{p}$, is determined according to the relative intensities of emission lines, $\mathrm{N}_{1}+\mathrm{N}_{2} / 4686 \mathrm{HeII}$ and $\mathrm{N}_{1}+N_{2} / \mathrm{H} \beta$. The excitation classes are obtained for $177 \mathrm{PN}$ with known distances and sizes of all classes - low $(\mathrm{p}=1-3)$, middle $(\mathrm{p}=4-8)$ and high $\left(\mathrm{p}=9-12^{+}\right)$. An empirical relationship between the excitation class $\mathrm{p}$ and the mean radius of the nebulae $R_{n}$ is discovered - the largest sizes occur for highly excited nebulae, and the smallest for low excitation ones. This relationship as well as excitation class $\mathrm{p}$, as an independent parameter admit an evolutionary interpretation (Gurzadyan, Egikyan 1991).

It is shown that after reaching the highest class of excitation $p=12^{+}$some part of nebulae again decreases their class of excitation with the further increases of sizes. The general diagram of this relationship, $\mathrm{p} \sim R_{n}$, has two nearly symetric branches - rising and descending with the apogee on $\mathrm{p}=12^{+}$. The whole number of nebulae in the descending part of this diagramm is more than an order smaller than their number in the rising part of the diagram.

The distribution of the number of PN versus the excitation class shows that the "Marathone" of nebulae from $p=1$ to $p=12^{+}$takes place with a rate too far to be constant: a nebula "lives" longer in the lowest excitation classes, $p=1-3$, after which very quickly gallops the classes $p=4-6$, and again the longer duration of life in classes $\mathrm{p} \sim 9-11$.

Moreover, there is also a tendancy that the mean expansion velocities increase when passing to high excitation nebulae.

The relationship $\mathrm{p} \sim R_{n}$ admits an evolutionary interpretation, and can be taken to some degree as an analogon of the Hertzsprung-Russel diagram for planetary nebulae.

At present we can establish that PN may be of low excitation class, from 1 to 6 , at their birth, and of classes from $12^{+}$up to $1-2$ in their destruction or disappearing period.
\end{abstract}

\section{References}

Gurzadyan G.A., Egikyan A.G. 1991, Astrophys.Space.Sci.,181,73 